\title{
Vena Cava and Left Renal Vein Variations and Association with Other Anomalies Including Horseshoe Kidney
}

\author{
Serife Leblebisatan ${ }^{1,{ }^{*}}$, Bozkurt Gulek ${ }^{1}$ and Gokhan Soker ${ }^{1}$ \\ ${ }^{1}$ Department of Radiology, Adana City Hospital, University of Health Sciences, Adana, Turkey \\ "Corresponding author: Department of Radiology, Adana City Hospital, University of Health Sciences, Dr. Mithat Ozhan Bulvarı/4522 Sok, Postal Code: 01060, Yuregir, Adana, \\ Turkey. Tel: +90-5058030860, Email: sefleblebi@gmail.com
}

Received 2018 May 21; Revised 2018 August 05; Accepted 2018 August 08.

\begin{abstract}
Objectives: The purpose of this study was to detect the types and prevalences of vena cava variations, as well as looking for gender differences and associating anomalies.

Patients and Methods: The computed tomography (CT) images of 5763 patients who had undergone thorax and abdominal CT examinations were evaluated retrospectively for superior vena cava (SVC), inferior vena cava (IVC), and left renal vein (LRV) variations. Results: Vena cava variations were detected in 637 (11\%) patients. SVC variations were present in $15(0.26 \%)$ patients, while $36(0.64 \%)$ patients had IVC, and 596 patients (10.34\%) had LRV variations. It was also shown that there was no difference between the prevalences of the variations in terms of gender difference. When associating anomalies were evaluated, it was seen that there was a significant increase in the risk of observing the horseshoe kidney anomaly in patients with retroaortic left renal vein (RALRV) variation.

Conclusion: Vena cava variations are not rare. When present, they may coexist with other vena cava variations or other variations. These variations can easily be detected on CT. Properly characterizing and classifying IVC variations is crucial for proper planning of surgical interventions and transvenous interventions to prevent serious complications and failures.
\end{abstract}

Keywords: Inferior Vena Cava (IVC), Left Renal Vein (LRV), Superior Vena Cava (SVC), Vena Cava

\section{Background}

Superior vena cava (SVC), inferior vena cava (IVC) and left renal vein (LRV) variations are not rare at all, and they have been diagnosed with an ever-increasing rate (1-4). Vena cava variations may stay asymptomatic throughout a lifetime (4). Knowing the presence of these variations will affect morbidity and mortality in patients undergoing operations and interventional procedures. So, it is very important that the radiologists be aware of these variations $(1,2,4,5)$.

Among the whole group of vena cava variations, there are some that are encountered rather more frequently, but the spectrum of variations is rather wide due to the complicated phases of embryologic development $(4,6)$. Despite the difficulties of categorization, there is a rather simple method of classification (Box 1$)$.

\section{Objectives}

The purpose of this study was to detect the types and prevalences of vena cava variations, as well as looking for gender differences and associating anomalies.

\begin{tabular}{l}
\hline Box 1. Classification of Vena Cava Variations \\
\hline Vena cava variations \\
\hline 1-SVC variations \\
Persistent left SVC \\
Double SVC \\
2- IVC variations \\
Left IVC \\
Double IVC \\
IVC interruption with azygos/hemiazygos continuation \\
Circumcaval ureter \\
3- LRV variations \\
Circumaortic LRV(CALRV) \\
Retroaortic LRV(RALRV) \\
\hline $\begin{array}{l}\text { Abbreviations: IVC, inferior vena cava; LRV, left renal vein; SVC, superior vena } \\
\text { cava. }\end{array}$
\end{tabular}

\section{Patients and Methods}

In this study, patients who underwent a thorax and an abdominal computed tomography (CT) in the same ses- 
sion were evaluated retrospectively. The patients belonged to the period between January 2015 and February 2016. Patients whose vena cava and LRV could not be evaluated due to motion artifacts, lymphadenopathy, insufficient contrast enhancement, and similar reasons, and patients with left nephrectomies were excluded from the study. The CT images of the remaining 5763 patients were then evaluated on the basis of the appearances of the SVC, IVC, LRV, ureters, and associated congenital malformations. The LRV variations were not considered as numeric variations; instead, they were categorized as being either a circumaortic left renal vein (CALRV) or a retroaortic left renal vein (RALRV). The CT examinations were performed in three CT scanners. Two of these scanners were GE Optima 520 s, and one was a Toshiba Alexion. All three were 16-detector systems. The GE Optimas were products of 2014, while the Toshiba Alexion had been produced in 2010. The CT examinations were performed by utilizing 5-mm slice thicknesses.

IBM SPSS (IBM Corp. Released 2011. IBM SPSS Statistics for Windows, Version 20.0. Armonk, NY: IBM Corp) pocket program was utilized for the statistical analyses. Categoric measurements were listed as numbers and percentages, while numeric measurements were laid as mean and standard deviation values (the median, and minimummaximum values, when necessary). The Chi-square test was utilized for the comparison of anomaly frequencies based on gender differences. In all statistical tests, the statistical significance level was appointed as 0.05 .

\section{Results}

Of the 5763 patients, 3594 (62\%) were males, and 2169 (38\%) were females. The ages of the patients varied between 1 day and 109 years (mean $\pm S D=47 \pm 20$ ). The median was 49 years. Vena cava variations were detected in 637 patients. SVC variations were present in 15 patients, whereas 36 patients demonstrated IVC and 596 patients demonstrated LRV variations. In the group of SVC variations, four patients demonstrated left SVC, while 11 demonstrated double SVC. In the group of IVC variations, there were left IVC in seven patients; double IVC in 27 patients, interruption of the IVC with azygos/hemiazygos continuation in two patients and a circumcaval ureter in one patient. In 11 of the 27 double IVC patients, an anastomosis was present at the level of the iliac veins. In one of the two IVC interruption patients, there was an azygos continuation and the azygos vein was draining both the two renal and two iliac veins. In the other patient, there were both azygos and hemiazygos continuation. The azygos vein was draining the right renal and right iliac veins; while the hemiazygos vein was draining the left renal and left iliac veins (Figure 1). In both patients, the hepatic veins were uniting and then draining into the right atrium. Of the 596 patients with LRV variations, 311 had CALRV, and 285 had
RALRV. The numbers, genders, and prevalences of the variations of the patients diagnosed with vena cava variations are given in Table 1.

There were other system variations in 115 patients and they are summarized in Table 2. Of these patients, 18 had vena cava variations. One of these patients demonstrated double IVC and an associating RALRV, while 10 had RALRV, and seven had CALRV variations (Table 3 ).

In ten patients, there were coexistences of vena cava variations (0.17\%). In one of these patients, double SVC + left IVC anomalies coexisted. Another one had left SVC + double IVC + RALRV variations (Figure 2). Six patients demonstrated double IVC + RALRV variations (Figure 3). One patient had double IVC + CALRV, while another had IVC interruption + RALRV.

\section{Discussion}

The prevalence of congenital vena cava and left renal vein variations have been reported to be between $0.07 \%$ and $8.7 \%$ in the literature (1). In our study, the prevalence of vena cava variations was found to be $0.8 \%$.

The persistent left SVC (PLSVC) is the location of an SVC in the left side, with or without the presence of a right SVC. In our study, the PLSVC prevalence was found to be $0.28 \%$. This prevalence is reported to be between $0.3 \%$ and $0.5 \%$ (7), and this is consistent with the results of our study. We found the prevalence of the isolated left SVC variation as $0.07 \%$, and that of the double SVC variation as $0.2 \%$, in our study. The isolated left SVC has been reported to be $20 \%$ in PLSVC patients, in the literature (8). This prevalence was found to be $25 \%$ in our study.

In the literature, the prevalence of IVC variations is reported to be $0.5 \%$ (6). In our study, this prevalence was found to be $0.6 \%$. The left IVC is seen to the left of the aorta at infrarenal level. The left IVC joins the left renal vein, which crosses anterior to the aorta. The left renal vein and the right renal vein units and the normal right sided prerenal IVC is formed. The left IVC prevalence has been reported in the literature to be between $0.2 \%$ and $0.5 \%(1,6$, $9,10)$. We found this prevalence as $0.1 \%$. Our result was lower than that reported in the literature, and this may contribute to the differences in patient populations. When infrarenal IVCs are seen on both sides of the aorta it is defined as the double IVC. The double IVC prevalence is reported to be between $0.2 \%$ and $0.3 \%$ in the literature $(1,2$, $6,9)$. In our study, this prevalence came out as $0.4 \%$, and it was a bit higher than the literature value. This, too, can be attributed to the differences in patient populations. Chen et al. have reported, after scanning the literature, that the chance of finding an interiliac vein in patients with a double IVC was $67.9 \%$ (11). In our study, we found this prevalence value as $40 \%$. The IVC interruption means that the hepatic segment of the IVC is absent. The suprarenal IVC 

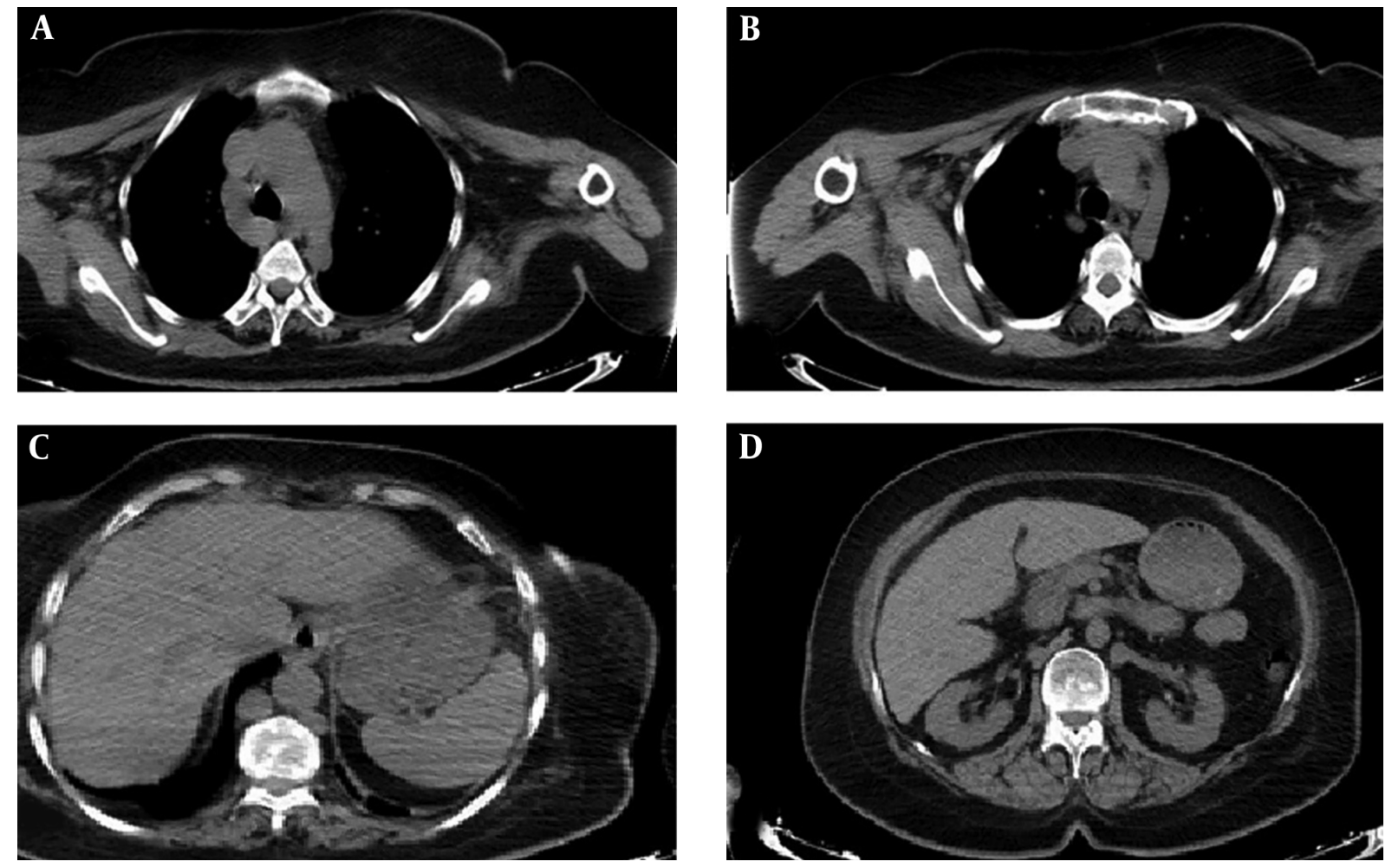

Figure 1. A 54-year-old female patient. Axial thorax and upper abdomen CT images without contrast show double inferior vena cava (IVC) interruption with azygos and hemiazygos continuation. The azygos vein drains into the superior vena cava (SVC) (A) while the hemiazygos vein drains into the brachiocephalic truncus via the accessory hemiazygos vein (B). Both the azygos and hemiazygos veins are dilated. The hepatic portion of the IVC cannot be seen (C). The azygos vein is seen to give the right renal vein and then continue as the right iliac vein. The hemiazygos vein, on the other hand, gives the left renal vein and continuous as the left iliac vein (D).

\begin{tabular}{|c|c|c|c|}
\hline VC variants & All patients $(n=5763)$, No. $(\%)$ & Male & Female \\
\hline VC variants (total) & $637(11)$ & 388 & 249 \\
\hline SVC variants & $15(0.26)$ & 9 & 6 \\
\hline Left SVC & $4(0.07)$ & 3 & 1 \\
\hline Double SVC & $11(0.19)$ & 6 & 5 \\
\hline IVC variants & $36(0.64)$ & 25 & 11 \\
\hline Left IVC & $7(0.12)$ & 4 & 3 \\
\hline Double IVC & $27(0.47)$ & 20 & 7 \\
\hline Double IVC with interiliac vein & $11(0.19)$ & 6 & 5 \\
\hline IVC interruptionwith azygos/hemiazygos continuation & $2(0.03)$ & 0 & 2 \\
\hline Circumcaval ureter & $1(0.017)$ & 1 & 0 \\
\hline LRV variants & $596(10.34)$ & 360 & 236 \\
\hline CALRV & $311(5.4)$ & 197 & 114 \\
\hline RALRV & $285(4.95)$ & 163 & 122 \\
\hline
\end{tabular}

Abbreviations: CALRV, circumaortic left renal vein; IVC, inferior vena cava; LRV, left renal vein; RALRV, retroaortic left renal vein; SVC, superior vena cava.

passes posterior to the diaphragmatic crura to enter the thorax as the azygos or hemiazygos vein or both. The literature reports the prevalence of the interruption of the
IVC with azygos/hemiazygos continuation as $0.6 \%(1,6,10)$. We found the prevalence of interruption of the IVC with azygos/hemiazygos continuation as $0.03 \%$. This was a way 


\begin{tabular}{|c|c|c|c|}
\hline Associating anomaly & All patients $(n=5763)$, No. $(\%)$ & Male & Female \\
\hline ARSA & $57(0.99)$ & 27 & 30 \\
\hline Renal anomalies & $40(0.69)$ & 29 & 11 \\
\hline Horseshoe kidney & $19(0.3)$ & 14 & 5 \\
\hline PAPVR & $10(0.17)$ & 5 & 5 \\
\hline Right arcus aorta & $4(0.07)$ & 2 & 2 \\
\hline Situs inversus totalis & $3(0.05)$ & 1 & 2 \\
\hline Left innominate vein anomaly & $1(0.017)$ & 0 & 1 \\
\hline Total & 115 (1.99) & 64 & 51 \\
\hline
\end{tabular}

Abbreviations: ARSA, aberrant right subclavian artery; PAPVR, partial anomalous pulmonary venous return.
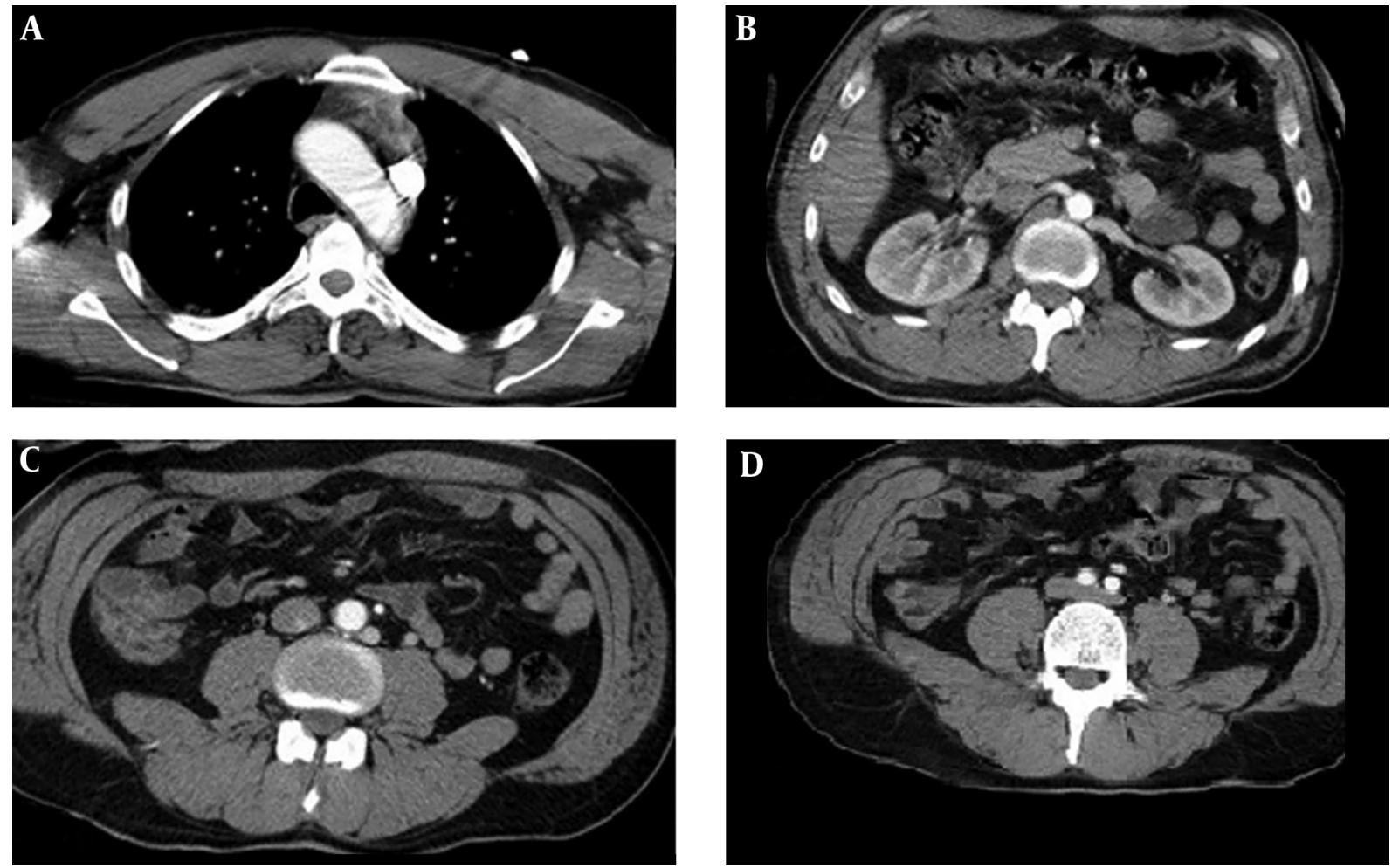

Figure 2. A 42-year-old male patient. Axial contrast enhanced thorax and upper abdomen images. There is a left superior vena cava SVC, but no right SVC (A). The hemiazygo vein which parts from the left SVC givesthe left renal vein (LRV) (B) and then continues downwards as a second IVC (C), finally uniting with the right inferior vena cava (IVC), through an anastomosis, at the level of the iliac veins (D).

lower than the literature value. The circumcaval ureter describes abnormal course of ureter. The proximal ureter courses posterior to the IVC, then emerges to the right of the aorta, coming to lie anterior to the IVC. The circumcaval ureter prevalence has been reported in the literature to be $0.07 \%$ in one article (1), and $0.1 \%$ in another (10). It has also been reported that the prevalence was 2.8 to 4 times higher in males than females (1). In our study, the prevalence of the circumcaval ureter anomaly was found to be $0.02 \%$, and the patient was a male.

The left renal vein variations includes retroaortic left renal vein (RALRV) and circumaortic left renal vein (CALRV) variations. The left renal vein normally passes anterior to the aorta. RALRV means that left renal vein passes posterior to the aorta. CALRV means that there are two left renal veins; one of them passes anterior to the aorta and the 

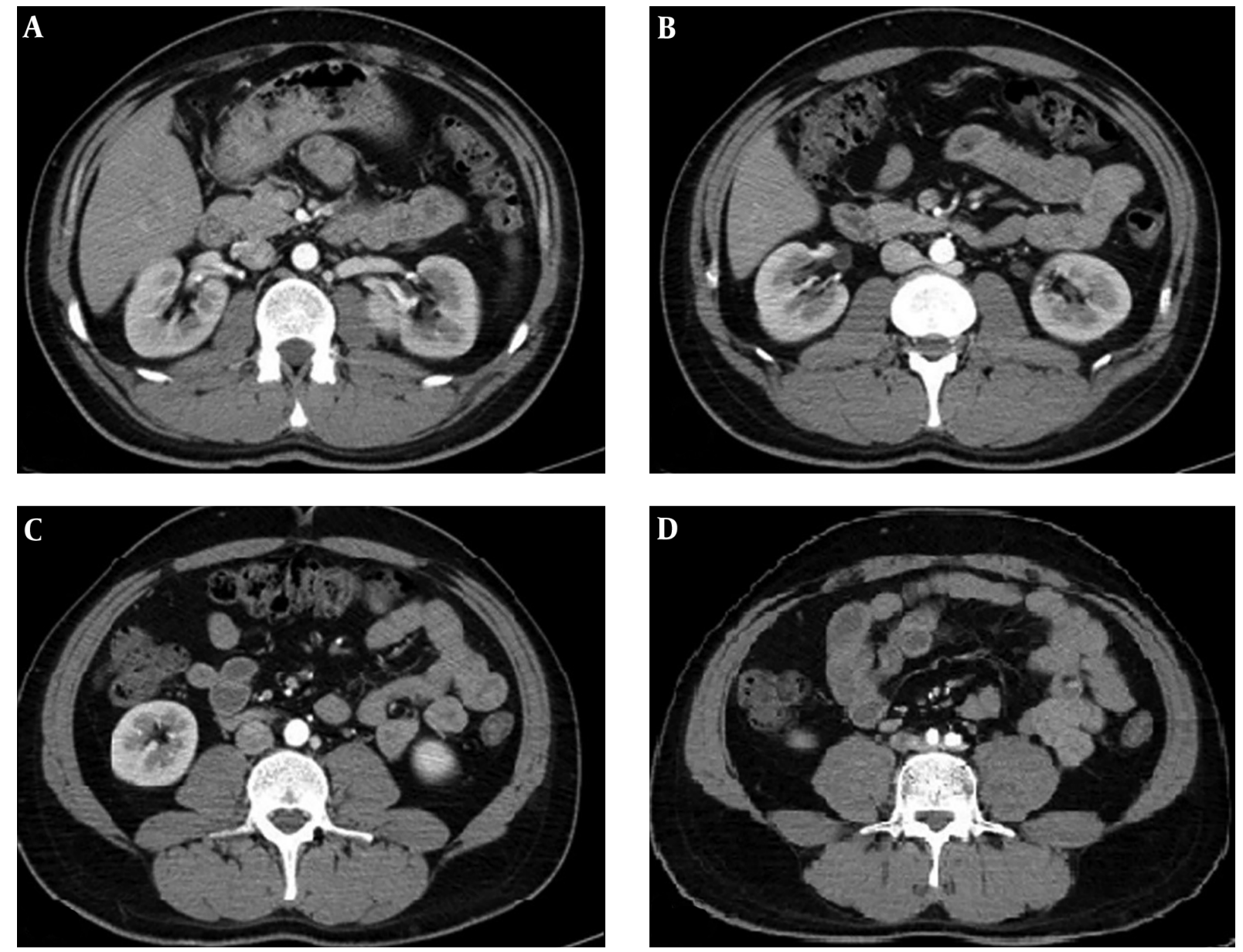

Figure 3. A 30-year-old male patient. The left renal vein (LRV) passes from the posterior aspect of the aorta and then drains into the right inferior vena cava (IVC) (A and B). Another branch goes directly downwards (C), finally anastomosing with the right IVC caudally, at the level of the iliac bifurcation (D).

other one posterior to the aorta. Koc et al. found the IVCRV variation prevalence to be $23 \%$ in their series of 1120 patients. The authors have found the prevalence of CALRV as $5.5 \%$, and that of RALRV as $4.6 \%$ (10). Sahin et al. found the prevalence of LRV anomalies as $2.3 \%$, that of RALRV as $2 \%$, and that of CALRV as $0.3 \%$ (12). Ayaz et al. reported the prevalence of LRV variations as 5.85\%, that of CALRV as 3.15\%, and that of RALRV as $2.70 \%$. These authors have also found that these prevalences were independent of gender difference (13). It has also been reported that CALRV is the most frequently encountered LRV variation (10). In our study, we found the prevalence of LRV variations as $10.34 \%$, the CALRV prevalence as $5.4 \%$, and the RALRV prevalence as $4.95 \%$. In our study, no statistically significant difference was found between genders, in terms of prevalences of both vena cava and left renal vein variations.

When the chance of coexistence of vena cava variations was studied, the prevalence of the coexistence of vena cava anomalies was found to be $0.17 \%$. The prevalence of encountering an association of SVC + IVC + LRV variations was found to be $0.017 \%$. We found the IVC and LRV association prevalence as $0.13 \%$. There is no study in the literature in which the association of multiple vena cava variations is evaluated, so our study is the first. Hence, vena cava variations may coexist. Some variations may be unanticipated and difficult to define.

When we studied other anomalies which might be coexisting with vena cava variations, we tried to evaluate the prevalences of these anomalies and find out if they demonstrated any differences between genders. Then we came to the following conclusions: We found the aberrant right subclavian artery (ARSA) prevalence in our study to be $0.989 \%$, which was accepted as $1 \%$. ARSA is a rare anomaly, in which the right subclavian artery arises from the aortic arch after the left subclavian artery instead of brachiocephalic truncus. The ARSA frequency is reported to be $1 \%$ 


\begin{tabular}{lc}
\hline Table 3. Association of Vena Cava Variations and Additional Anomalies & \\
\hline Vena cava variation & No. \\
\hline SVC variants & 0 \\
\hline IVC variants & \\
\hline \multicolumn{2}{c}{ Double IVC with interiliac vein + RALRV } \\
\hline Right malrotationed ectopic kidney & 1 \\
\hline LRV variants & \\
\hline CALRV & \\
\hline ARSA & 4 \\
\hline Horseshoe kidney & 1 \\
\hline Right renal ectopia & 1 \\
\hline Right sided arcus aorta & 1 \\
\hline RALRV & \\
\hline Horseshoe kidney & 4 \\
\hline ARSA & 3 \\
\hline Left malrotationed kidney & 2 \\
\hline Situs inversus totalis & 1 \\
\hline
\end{tabular}

Abbreviations: ARSA, aberrant right subclavian artery; CALRV, circumaortic left renal vein; IVC, inferior vena cava; LRV, left renal vein; RALRV, retroaortic left renal vein; SVC, superior vena cava.

in the general population and is considered to be the most frequent aortic arc anomaly (14). Our finding was in congruence with the literature. Karacan et al. have found that the ARSA incidence rate was higher in women than in men. These values were $2.5 \%$ for women, and $0.5 \%$ for men (15). In our study, the prevalence was $1.38 \%$ for women, whereas it was $0.75 \%$ in men.

The partial anomalous pulmonary venous return (PAPVR) is a rare anomaly in which some of the pulmonary veins drain into the systemic circulation, not into the left atrium. PAPVR anomaly rate reported as $0.1 \%$ in Ho et al. study (16), whereas it was reported to be $0.2 \%$ in the study of Haramati et al. (17). In our study, the PAPVR incidence was found to be $0.17 \%$, and this was in line with the literature.

The right-sided aortic anomaly means that the aortic arch lies to the right of the trachea. Rea et al. have found the right aortic arch anomaly prevalence to be $0.5 \%$ (18). We found the right aortic arch anomaly prevalence to be $0.07 \%$, and this was significantly lower than that reported in the literature.

All the internal organs positioned at the opposite side with dextrocardia is termed situs inversus totalis. Its prevalence is reported in the literature to be between $1 / 5000$ and $1 / 20000$ (19). We found this prevalence as $0.05 \%$, or $1 / 2000$, in our study. This value was higher than reported in the literature.

Yamamuro et al. reported the prevalence of the left brachiocephalic vein anomaly to be between $0.03 \%$ and $0.062 \%$ (20). Our result was $0.017 \%$, and lower than the literature. Some prevalences that we found to be different than those reported in the literature belonged to the left innominate vein, right aortic arch, and situs inversus totalis, abnormalities. This may be due to the difference in patient populations.

In our study, we found no increase in the incidence of other anomalies detected by CT in patients with SVC, IVC and CALRV variations. With the presence of RALRV variation, the risk of encountering a renal anomaly and horseshoe kidney anomaly was found to increase 4.16 and 5.18 fold, respectively.

It can be seen that the coexistence of vena cava or LRV anomalies with the horseshoe kidney anomaly have been reported in the literature. The horseshoe kidney is the most common type of renal fusion anomaly. The horseshoe kidney anomaly prevalence has been reported in the literature to be between $0.1 \%$ and $0.3 \%$ (7). We found this prevalence as $0.3 \%$, and this was in line with the literature. Ichikawa et al. performed a study in which they examined the incidence of SVC anomalies in patients with and without horseshoe kidney abnormalities, and compared these two groups. This study revealed $4.2 \%$ incidence of SVC variations in patients with horseshoe kidney abnormalities and $0.22 \%$ incidence in patients without this abnormality $(\mathrm{P}<0.001)$ (21). Jaffer and Chandiramani reported a case of coexisting double SVC and horseshoe kidney(7). In none of the patients with the horseshoe kidney abnormality in our study was there an associating SVC variation.

Ichikawa et al. reported that IVC anomalies had been encountered significantly more frequently in patients with kidney disease than the ones without (22). The same authors also reported in another study that the frequency of IVC variations had been found to be significantly higher in patients with the horseshoe kidney anomaly, in comparison to the normal population (5.7\%) (23). On the other hand, Smith and Frost reported a coexistence between IVC anomalies and a horseshoe kidney in two patients (24). In our study, we did not detect any IVC anomaly in patients with horseshoe kidney.

Ichikawa et al. mentioned that they evaluated patients with horseshoe kidneys for major venous anomalies and found out that the prevalence of renal vein anomalies was no different than that of the normal population $(28.6 \%)$ (23). In our study, RALRV variation was found to be higher in patients with horseshoe kidneys, in comparison to those patients without horseshoe kidneys. Our results showed that the chance of seeing a RALRV was $26.3 \%$ in patients with horseshoe kidneys, while this number was found to be $4.9 \%$ in patients without the horseshoe kidney anomaly. Thus, the risk of encountering a RALRV was found to be 5.18 times higher in patients with horseshoe kidneys, in comparison to those patients without horseshoe kidneys. 
In conclusion, vena cava variations may present with an astonishingly wide spectrum of appearances. These variations may coexist, or they may exist in association with other variations. We would like to conclude that both the radiologist and the clinician must be alerted for the possible existence and coexistence of these variations, in terms of operational and interventional procedural safety, morbidity and mortality.

\section{Acknowledgments}

The authors thank Ilker Unal (Department of Biostatistics, Cukurova University Hospital, Adana) for his assistance with the statistical analysis in this study.

\section{Footnotes}

Authors' Contributions: Serife Leblebisatan developed the original idea and the protocol, abstracted and analyzed data and wrote the manuscript. Bozkurt Gulek and Gokhan Soker contributed to the development of the protocol, abstracted data, and prepared the manuscript.

Financial Disclosure: Authors indicating that they have no financial interests related to the material in the manuscript.

Funding/Support: Authors indicate that they have no funding/support related to the manuscript.

Ethical Considerations: The Ethical Committee of the University of Cukurova provided statements regarding the ethical acceptability of this work.

\section{References}

1. Kellman GM, Alpern MB, Sandler MA, Craig BM. Computed tomography of vena caval anomalies with embryologic correlation. $R a-$ diographics. 1988;8(3):533-56. doi: 10.1148/radiographics.8.3.3380993. [PubMed: 3380993].

2. Bass JE, Redwine MD, Kramer LA, Huynh PT, Harris JH. Spectrum of congenital anomalies of the inferior vena cava: Cross-sectional imaging findings. Radiographics. 2000;20(3):639-52. doi: 10.1148/radiographics.20.3.g00ma09639. [PubMed:10835118].

3. Yang C, Trad HS, Mendonca SM, Trad CS. Congenital inferior vena cava anomalies: A review of findings at multidetector computed tomography and magnetic resonance imaging. Radiol Bras vol. 2013;46(4). doi: 10.1590/s0100-39842013000400009.

4. Qian ZY, Yang MF, Zuo KQ, Cheng J, Xiao HB, Ding WX. Computed tomography manifestations of common inferior vena cava dysplasia and its clinical significance. Exp Ther Med. 2013;5(2):631-5. doi: 10.3892/etm.2012.830. [PubMed: 23407792]. [PubMed Central: PMC3570192].

5. Boyaci N, Karakas E, Dokumaci DS, Yildiz S, Cece H. Evaluation of left renal vein and inferior vena cava variations through routine abdominal multi-slice computed tomography. Folia Morphol (Warsz). 2014;73(2):159-63. doi: 10.5603/FM.2014.0017. [PubMed: 24902094].

6. Petik B. Inferior vena cava anomalies and variations: imaging and rare clinical findings. Insights Imaging. 2015;6(6):631-9. doi: 10.1007/s13244-015-0431-z. [PubMed: 26373648]. [PubMed Central: PMC4656244].
7. Jaffer F, Chandiramani V. Concomitant persistent left superior vena cava and horseshoe kidney. Case Rep Nephrol. 2015;2015:178310. doi: 10.1155/2015/178310. [PubMed: 25664191]. [PubMed Central: PMC4309029].

8. Gonzalez-Juanatey C, Testa A, Vidan J, Izquierdo R, Garcia-Castelo A, Daniel C, et al. Persistent left superior vena cava draining into the coronary sinus: Report of 10 cases and literature review. Clin Cardiol. 2004;27(9):515-8. [PubMed: 15471164].

9. Kandpal H, Sharma R, Gamangatti S, Srivastava DN, Vashisht S. Imaging the inferior vena cava: A road less traveled. Radiographics. 2008;28(3):669-89. doi: 10.1148/rg.283075101. [PubMed: 18480478].

10. Koc Z, Ulusan S, Oguzkurt L, Tokmak N. Venous variants and anomalies on routine abdominal multi-detector row CT. Eur J Radiol. 2007;61(2):267-78. doi: 10.1016/j.ejrad.2006.09.008. [PubMed: 17049792].

11. Chen H, Emura S, Nagasaki S, Kubo KY. Double inferior vena cava with interiliac vein: A case report and literature review. Okajimas Folia Anat Jpn. 2012;88(4):147-51. [PubMed: 22645906].

12. Sahin C, Kacira OK, Tuney D. The retroaortic left renal vein abnormalities in cross-sectional imaging. Folia Med (Plovdiv). 2014;56(1):38-42. [PubMed: 24812921].

13. Ayaz S, Ayaz UY. Detection of retroaortic left renal vein and circumaortic left renal vein by PET/CT images to avoid misdiagnosis and support possible surgical procedures. Hell J Nucl Med. 2016;19(2):135-9. doi: 10.1967/s002449910367. [PubMed: 27331207].

14. Tzilas V, Koti A, Tsoukalas G. A rare cause of dysphagia-aberrant right subclavian artery (ARSA). Eur Rev Med Pharmacol Sci. 2009;13(4):313-6. [PubMed: 19694347].

15. Karacan A, Turkvatan A, Karacan K. Anatomical variations of aortic arch branching: Evaluation with computed tomographic angiography. Cardiol Young. 2014;24(3):485-93. doi: 10.1017/S1047951113000656. [PubMed: 23694814].

16. Ho ML, Bhalla S, Bierhals A, Gutierrez F. MDCT of partial anomalous pulmonary venous return (PAPVR) in adults. J Thorac Imaging. 2009;24(2):89-95. doi: 10.1097/RTI.0b013e318194c942. [PubMed: 19465830].

17. Haramati LB, Moche IE, Rivera VT, Patel PV, Heyneman L, McAdams $\mathrm{HP}$, et al. Computed tomography of partial anomalous pulmonary venous connection in adults. J Comput Assist Tomogr. 2003;27(5):743-9. [PubMed: 14501365].

18. Rea G, Valente T, Iaselli F, Urraro F, Izzo A, Sica G, et al. Multi-detector computed tomography in the evaluation of variants and anomalies of aortic arch and its branching pattern. Ital J Anat Embryol. 2014;119(3):180-92. [PubMed: 26749678].

19. Uludag M, Yetkin G, Kartal A. Single-incision laparoscopic cholecystectomy in situs inversus totalis. JSLS. 2011;15(2):239-43. doi: 10.4293/108680811X13071180407032. [PubMed: 21902984]. [PubMed Central: PMC3148880].

20. Yamamuro H, Ichikawa T, Hashimoto J, Ono S, Nagata Y, Kawada S, et al. Congenital anomalies of the left brachiocephalic vein detected in adults on computed tomography.Jpn J Radiol. 2017;35(10):597-605. doi: 10.1007/s11604-017-0673-4. [PubMed: 28849388].

21. Ichikawa T, Sekiguchi T, Kawada S, Koizumi J, Endo J, Yamada Y, et al. Study of the association between an anomalous superior vena cava and horseshoe kidney. Circ J. 2012;76(5):1253-8. [PubMed: 22343193].

22. Ichikawa T, Kawada S, Koizumi J, Endo J, Itou C, Matsuura K, et al. Anomalous inferior vena cava associated with horseshoe kidney on multidetector computed tomography. Clin Imaging. 2013;37(5):88994. doi: 10.1016/j.clinimag.2013.03.005. [PubMed: 23849103].

23. Ichikawa T, Kawada S, Koizumi J, Endo J, Iino M, Terachi T, et al. Major venous anomalies are frequently associated with horseshoe kidneys. Circ J. 2011;75(12):2872-7. [PubMed: 22001291].

24. Smith TR, Frost A. Anomalous inferior vena cava associated with horseshoe kidneys. Clin Imaging. 1996;20(4):276-8. [PubMed: 8959368]. 